\title{
Förster energy transfer between neighbouring chromophores in C-phycocyanin trimers
}

\author{
Tomas Gillbro a , Andrei V. Sharkov ${ }^{\mathrm{b}}$, Igor V. Kryukov ${ }^{\mathrm{b}}$, Eugeny V. Khoroshilov ${ }^{\mathrm{b}}$, \\ Piotr G. Kryukov ${ }^{\mathrm{b}}$, Richard Fischer ${ }^{\mathrm{c}}$ and Hugo Scheer ${ }^{\mathrm{c}}$ \\ a Department of Physical Chemistry, University of Umeå, Umeå (Sweden), \\ ${ }^{b}$ P.N. Lebedev Physics Institute, Russian Academy of Sciences, Moscow (Russia) \\ and ${ }^{c}$ Botanisches Institut der Universität München, München (Germany)
}

(Received 2 June 1992)

Key words: Phycocyanin; Excitation energy transfer; Femtosecond spectroscopy; Anisotropy; Photosynthesis

The excitation-energy transfer in C-phycocyanin (C-PC) trimers and monomers isolated from phycobilisomes of Mastigocladus laminosus has been studied by polarization femtosecond laser spectroscopy. Excitation with 70 -fs pulses at $615 \mathrm{~nm}$ gave rise to a 500 -fs energy-transfer process that was observed only in trimeric preparations. The rate of the process is in agreement with earlier calculated Förster energy transfer rates between neighbouring $\alpha-84$ and $\beta-84$ chromophores of different monomeric subunits. This process is most clearly seen in the anisotropy decay kinetics. As a result of femtosecond excitation-energy transfer, the anisotropy relaxes from 0.4 to 0.23 . The final anisotropy value is in fair agreement with the results of calculations based on the crystal structure and spectroscopic data of C-PC trimers. Our results support the conclusion that Förster energy transfer can occur between excitonically coupled chromophores.

\section{Introduction}

Cyanobacteria are among the oldest oxygen-evolving photosynthetic organisms [1] and they are believed to be relatives or even ancestors of algal and higher plant chloroplasts. In order to collect the environmental solar energy flux in an efficient manner, their photosynthetic apparatus contains large amounts of membrane-attached light harvesting complexes, the so-called phycobilisomes. These antennas, which they share with red algae, distinguish cyanobacteria from other algae and green plants. They are comprised of biliproteins containing covalently linked bile pigment chromophores and of (mainly) chromophore-less linker polypeptides. Some of the common chromoproteins are C-phycocyanin (C-PC), allophycocyanin (APC), phycoerythrin (PE) and phycoerythrocyanin (PEC) [2,3]. APC is contained in the core of the phycobilisome and is closest to Photosystem II in the thylakoid membrane [2]. The rods radiating from the core are composed of

Correspondence to: T. Gillbro, Department of Physical Chemistry, University of Umeå, S-90187 Umeå, Sweden.

Abbreviations: C-PC, C-phycocyanin; APC, allophycocyanin; CPM, colliding pulse-mode-locked.
C-PC (proximal) and in many species also of PE and PEC [2].

The basic building blocks of the phycobilisomes are trimeric units of the different chromoproteins. In C-PC, the two subunits ( $\alpha$ and $\beta$ ) contain one $\alpha(\alpha-84)$ ant two $\beta$ chromophores $(\beta-84, \beta-155)$, respectively. These form a monomeric unit $(\alpha \beta)$, which is assembled into larger torus-shaped aggregates (trimers and hexamers). The other biliproteins are structured in a similar way, but contain a different number and often also different types of chromophores.

The structures of C-PC trimers or hexamers from three cyanobacteria are known from $X$-ray crystallography [4-6]. The structures and positions of the $\beta-155$, $\beta-84$ and $\alpha-84$ chromophores are highly conserved in different C-PC. In C-PC trimers (and hexamers) the closest distance, $20.8 \AA$, is found between $\alpha-84$ and $\beta-84$ chromophores of two neighbouring monomer units [5].

Previous absorption and fluorescence picosecond work on C-PC trimers has shown that the fastest energy transfer rates are in the order of $10-50$ ps [7-13]. A 23-ps component in C-PC trimers was originally interpreted as energy transfer from the donor chromophore $(\beta-155)$ to $\beta-84$ and $\alpha-84$ ones [7-12]. More recently, the same component was, however, suggested to play a part in a relaxation between two excitonic 
bands formed by an interaction between the closelysituated $\alpha-84$ and $\beta-84$ chromophores [13]. Indication of a sub-picosecond signal in C-PC trimers has also been reported [14].

Femtosecond analysis of energy transfer processes in APC trimers was reported in our previous publications $[15,16]$. The aim of this work was to study the energy transfer between the $\alpha-84$ and $\beta-84$ chromophores in C-PC trimers by femtosecond polarization techniques and to investigate whether the transfer can be best described by the Förster mechanism or by relaxation between excitonic states.

\section{Materials and Methods}

The C-PC trimers of Mastigocladus laminosus were prepared as described in Refs. 17, 18. The preparations were kept frozen until use. Before measurements, the preparations were diluted $(1: 3)$ into $0.1 \mathrm{M}$ phosphate buffer $(\mathrm{pH} 7)$. The monomers were prepared by adding NaSCN to $1.2 \mathrm{M}$ directly before the measurements [18].

All measurements were made at $20^{\circ} \mathrm{C}$ in a rotating cell of $1 \mathrm{~mm}$ optical path length. The absorbances of the samples at the excitation wavelength $(615 \mathrm{~nm})$ were 0.5 for monomers and trimers.

70 -femtosecond pulses at $615 \mathrm{~nm}$ from a CPM-laser were amplified at a $10 \mathrm{kHz}$ repetition-rate in a multipass jet amplifier [19]. These $0.5 \mu \mathrm{J}$ pulses are split and delivered to pump and probe channels $(10: 1)$ and the probe pulses are further split into probe and reference channels $(1: 1)$. After passing a stepping-motor-driven variable delay line, the probe beam is directed parallel to the pump beam, and both pulses are focused by a lens $(f=10 \mathrm{~cm})$ into a rotating cell. The pump beam has a linear polarization. A $\lambda / 4$ plate is inserted into the probe beam before the cell to produce circularly polarized light. A polarization of 0,90 and $54.7^{\circ}$ (magic angle) to the polarization of the pump beam is obtained with a polarizer in the probe beam. The energy of each pulse of the probe (after the sample cell) and reference beams is measured with photodiodes and stored in two channels of a CAMAC data acquisition system controlled by an IBM/PC computer. After storage of 100 probe and reference pulses with the sample excited, the excitation beam is blocked with a CAMAC-controlled shutter and 100 probe and reference pulses without excitation are stored. These data are enough to calculate the photoinduced absorbance change. At a fixed delay between pump and probe pulses, this procedure is repeated $50-100$ times and the average absorbance change is calculated. The delay is then changed with the CAMAC-controlled stepping motor and the $\Delta A(t)$-dependence is measured for $\|$, $\perp$ or magic angle polarization of the probe light. The

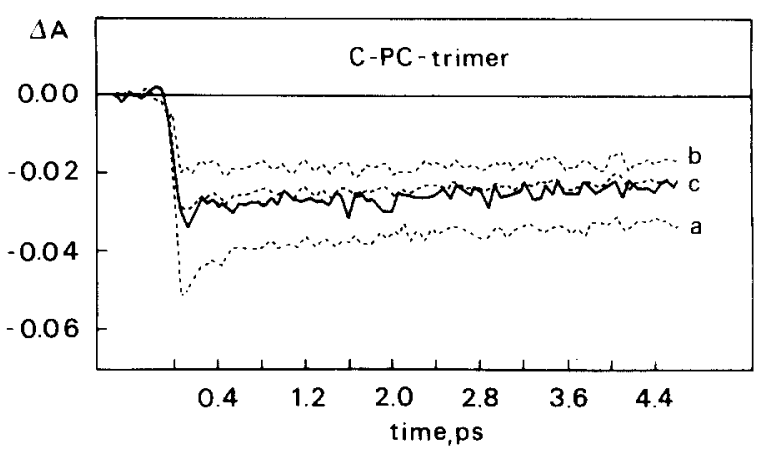

Fig. 1. Photoinduced absorbance changes at $615 \mathrm{~nm}$ measured for C-PC trimers with probe pulse polarization parallel (dotted line, a), perpendicular (dotted line, b) or at magic angle (solid line) to the polarization of the exciting pulse. Dotted line (c) shows isotropic decay calculated from traces (a) and (b)

anisotropy $r(t)$ and isotropic optical changes $\Delta A^{\text {iso }}(t)$ are then calculated according to the standard formulas:

$r(t)=\left(\Delta A^{\|}(t)-\Delta A^{\perp}(t)\right) /\left(\Delta A^{\|}(t)+2 \Delta A^{\perp}(t)\right)$

$\Delta A^{\text {iso }}(t)=\left(\Delta A^{\|}(t)+2 \Delta A^{\perp}(t)\right) / 3$

\section{Results}

Fig. 1 shows the absorbance changes measured with trimers of C-PC. The results obtained with probe pulse polarization at magic angle (solid line), parallel (a) or perpendicular (b) to the polarization of the exciting pulse are presented, as well as the isotropic signal (c) calculated from traces (a) and (b). The corresponding anisotropy calculated from traces (a) and (b) is shown in Fig. 2 and can be fit by a single exponential decay with $\tau=0.5 \pm 0.1 \mathrm{ps}$. Immediately after excitation, the anisotropy is equal to 0.4 and decays to the level of $0.23 \pm 0.02$ within the investigated time interval. To analyse the initial part of the decay kinetics, we have measured the absorbance changes within a 1-ps period in separate experiments (Fig. 3) and the absorption

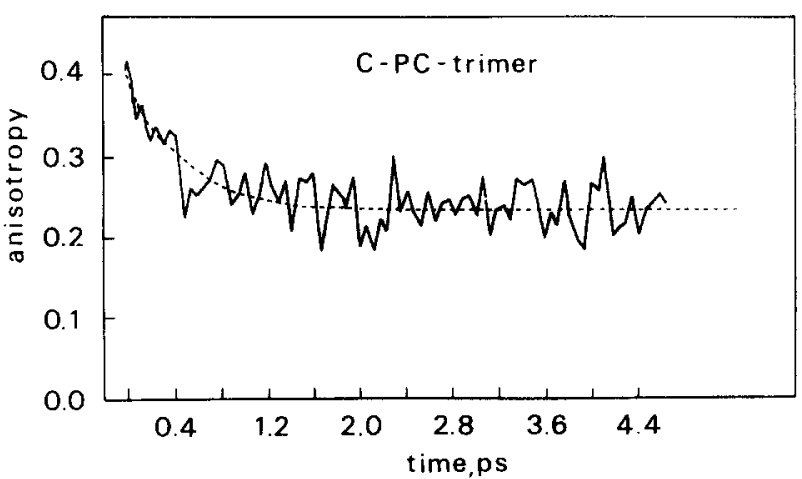

Fig. 2. Anisotropy decay calculated from curves (a) and (b) presented in Fig. 1. (solid line). Dotted line shows 0.5-ps sinle-exponential fit of this decay. 


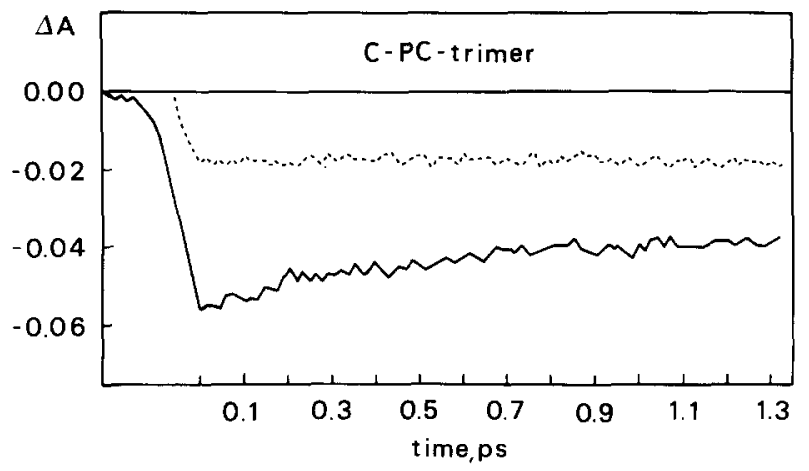

Fig. 3. The subpicosecond kinetics of C-PC trimers shown in detail. Solid line is for parallel and dotted line is for perpendicular polarization of the probing pulse, respectively.

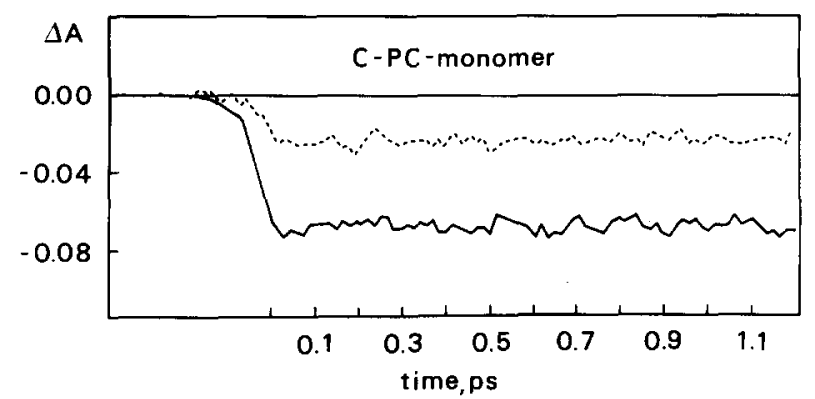

Fig. 4. The subpicosecond kinetics of C-PC monomers at $615 \mathrm{~nm}$. Parallel and perpendicular polarization of the probing pulse as in Fig. 3.

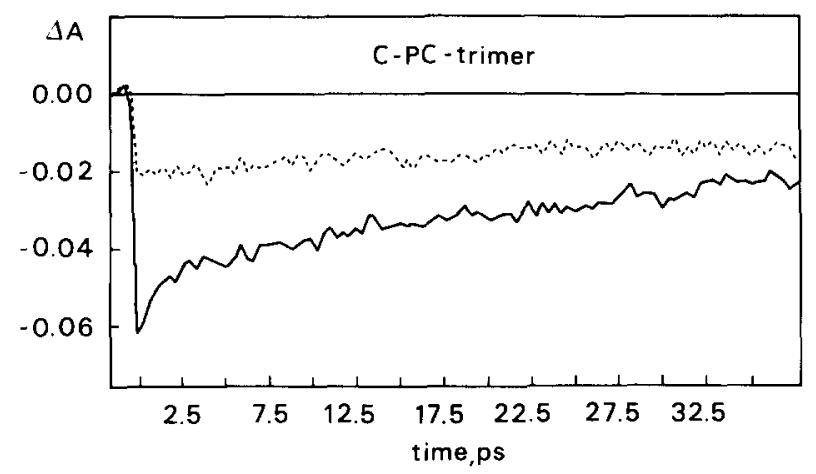

Fig. 5. The picosecond kinetics of C-PC trimers measured at $615 \mathrm{~nm}$ with parallel (solid line) and perpendicular (dotted line) polarization of the probing pulse.

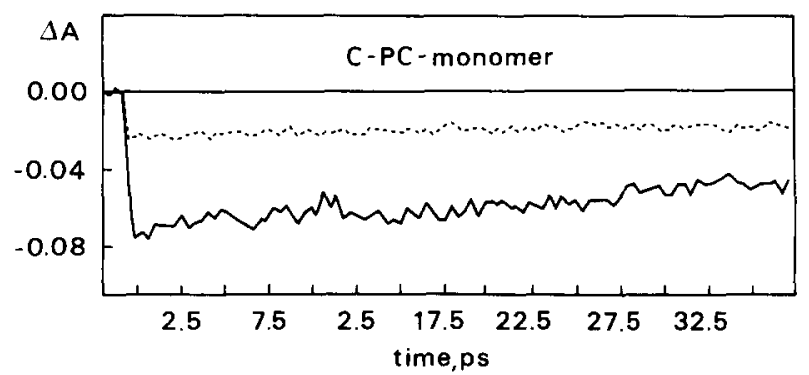

Fig. 6. The picosecond kinetics of C.PC monomers at $615 \mathrm{~nm}$. Parallel and perpendicular polarization of the probing pulse as in Fig. 5. recovery is estimated to be single-exponential with $\tau=540 \pm 50$ fs (parallel polarization).

The same time-scale is used in Fig. 4 to show C-PC monomer absorbance changes. No evident decay kinetics were obtained during the first picosecond after excitation for both polarizations of the probing pulse. To be sure that our results correspond to earlier picosecond investigations, we have measured the photoinduced absorbance changes during 30 ps after excitation for both C-PC trimers and monomers (Figs. 5 and 6 , respectively). One can see a slow picosecond decay for both preparations. The corresponding lifetimes are roughly estimated to be $30-100 \mathrm{ps}$ (in trimers, this picosecond decay follows the femtosecond one). In both preparations, the picosecond kinetics are observed only for parallel probe-pulse polarization and, therefore, are related to anisotropy decay (not shown).

In all figures, the zero point on the time scale $(t=0)$ is arranged close to the maximum absorption bleaching, rather than at the point of coincidence of pump and probe pulse maxima. At $t>0$ absorption recovery and anisotropy decay are exponential, and deconvolution is not required. At $t<0$, the anisotropy calculated from $\Delta A_{\|}(t)$ and $\Delta A_{\perp}(t)$ exhibits high noise level because of fast absorbance changes at the moment of excitation (Fig. 1) and this is the reason why it is not shown in Fig. 2.

\section{Discussion}

The anisotropy-recovery kinetics with a lifetime of about 0.5 ps were earlier observed in APC trimers and attributed to excitation-energy transfer between $\alpha-84$ and $\beta-84$ chromophores with different absorption spectra $[15,16]$. The most pronounced difference between the results obtained with APC and C-PC trimers is that femtosecond anisotropy decay was observed only for the C-PC trimers. This decay obviously is related to the interaction between neighbouring $\alpha-84$ and $\beta-84$ chromophores located in different monomers, because such a fast anisotropy decay was not recorded for C-PC monomers. For the interpretation of our results, the information about the distance and mutual orientation between the chromophores can be taken into account [5], as well as the individual spectra of the $\alpha-84, \beta-84$ and $\beta-155$ chromophores [20]. The spectrum of the $\alpha-84$ chromophore has been determined by Siebzehnrübl et al. [21] on the isolated $\alpha$-subunit and the spectra of the $\beta$-chromophores were derived from a curve resolution of the absorption spectrum of the isolated $\beta$-subunit [22]. From these data, Förster energy-transfer rates for different pairs of chromophores in C-PC trimers including the nearest pairs of $\alpha-84$ and $\beta-84$ chromophores have been calculated $[20,23]$. According to X-ray crystallography data the distance between chromophores in these pairs is $20.8 \AA$ and the 
angle between the chromophores is $65^{\circ}$ [5]. The rate of the downhill $\alpha-84 \rightarrow \beta-84$ transfer was calculated as $1530 \mathrm{~ns}^{-1}$ and that of the back reaction as $1160 \mathrm{~ns}^{-1}$ [23].

Rate equations for donor and acceptor excited-state populations, neglecting relaxation to the ground state, can be written as:

$\mathrm{d} N_{\mathrm{D}}^{*}(t) / \mathrm{d} t=-K_{\mathrm{DA}} N_{\mathrm{D}}^{*}(t)+K_{\mathrm{AD}} N_{\mathrm{A}}^{*}(t)$

$N_{\mathrm{D}}^{*}(t)+N_{\mathrm{A}}^{*}(t)=N^{*}$

where $K_{\mathrm{DA}}$ and $K_{\mathrm{AD}}$ are the rates of downhill and back reactions, respectively. $N_{\mathrm{D}}^{*}(t)$ and $N_{\mathrm{A}}^{*}(t)$ are the corresponding excited-state donor and acceptor populations, respectively, and $N^{*}=\mathrm{N}_{\mathrm{D}}^{*}(0)$ is determined by the initial conditions. The solution of Eqn. 2 for donor and acceptor excited-state populations is then:

$$
\begin{aligned}
N_{\mathrm{D}}^{*}(t)= & \left(N_{\mathrm{D}}^{*}(0)-K_{\mathrm{AD}} N^{*} /\left(K_{\mathrm{AD}}+K_{\mathrm{DA}}\right)\right) \mathrm{e}^{-\left(K_{\mathrm{AD}}+K_{\mathrm{DA}}\right) t} \\
& +K_{\mathrm{AD}} N^{*} /\left(K_{\mathrm{AD}}+K_{\mathrm{DA}}\right) \\
N_{\mathrm{A}}^{*}(t)= & N^{*}-N_{\mathrm{D}}^{*}(t)
\end{aligned}
$$

The experimentally observed kinetics should accordingly be determined by the sum of the rate constants and from the calculated rates, a lifetime of $370 \mathrm{fs}$ is expected. We can see that this lifetime calculated by the Förster mechanism is in reasonable good agreement with the 500-fs anisotropy relaxation time observed in this work (Fig. 2).

Since the energy transfer takes place between chromophores with different orientations, it is expected that the anisotropy will change during the excitationenergy equilibration between the two chromophores. We will show next that the final level of 0.23 for the measured anisotropy is in good agreement with the estimated $65^{\circ}$ angle between donor and acceptor transition dipole orientations calculated from the X-ray data [5].

Upon excitation at $615 \mathrm{~nm}$, all three types of chromophores are excited and the initial excited-state populations are proportional to the corresponding absorption cross sections at this wavelength, which are about $1.7: 1: 1$ for $\alpha-84, \beta-84$ and $\beta-155$, respectively. Initially, excited $\beta-155$ chromophores are not expected to undergo energy transfer during the first few picoseconds [7-12], which is also corroborated by the lack of this component for C-PC monomers. The population of excited $\alpha-84$ (donors) will change with time due to fast transfer of energy to $\beta-84$ according to Eqn. 3 . The final population is $N_{\mathrm{D}}^{*}(\infty)=\gamma N_{\mathrm{D}}^{*}(0)$, where $\gamma=$ $K_{\mathrm{AD}} /\left(K_{\mathrm{AD}}+K_{\mathrm{DA}}\right)=0.43$, and $N_{\mathrm{A}}^{*}(\infty)=(1-$ $\gamma) N_{\mathrm{D}}^{*}(0)$. For initially excited $\beta-84$ donors similar equations will hold also for the back reaction.

In the presence of several ensembles of excited molecules, the experimentally measured anisotropy is described as follows:

$r(t)=\frac{\sum_{i} r_{i}(t) \Delta A_{i}^{\mathrm{iso}}(t)}{\sum_{i} \Delta_{i}^{\mathrm{iso}}(t)}$

where $r_{i}(t)$ and $\Delta A_{i}^{\text {iso }}(t)$ are defined according to Eqn. 1 from $\Delta A_{i}^{\|}(t)$ and $\Delta A_{i}^{\perp}(t)$, which are absorbance changes due to $i^{\text {th }}$ ensemble. We also have $\Delta A^{\text {iso }}(t)=$ $\sum_{i} \Delta A_{i}^{\text {iso }}(t)$.

Assuming that the excited state absorption is very small at $615 \mathrm{~nm}$ for all chromophores, or $\sigma_{i}{ }^{*} \ll \sigma_{i}$, where $\sigma_{i}$ is the isotropic absorption cross-section at $615 \mathrm{~nm}$ for ground-state absorption and $\sigma_{i}{ }^{*}$ is that for excited state absorption, it follows that $\Delta A_{i}^{\text {iso }}(t)=$ $l \sigma_{i} N_{i}^{*}(t)$, where $l$ is the optical path length of the cell. The final anisotropy can now be written as:

$r(\infty)=\frac{\sum_{i} r_{i}(\infty) \sigma_{i} N_{i}^{*}(\infty)}{\sum_{i} \sigma_{i} N_{i}^{*}(\infty)}$

In our case, five ensembles contribute to Eqn. 5, namely initially excited $\beta-155$, initially excited $\alpha-84$ and $\beta-84$ (donors) and the same chromophores as acceptors. The excited-state populations $N_{i}^{*}(\infty)$ can be expressed from the initial excited-state populations created by the laser-exciting-pulse as mentioned above. The initial excited-state populations are in turn proportional to the corresponding ground-state absorption cross-sections and ground-state populations for ensembles which are excited by the laser pulse, the latter being equal in our case. To determine $r_{i}(\infty)$, we have to take into consideration that $r_{i}$ is a constant for each of the five ensembles of excited chromophores, assuming that only excitation energy-transfer processes influence the $r(t)$ value. The $r_{i}$ values are 0.4 for all initially-excited (donor) chromophores. The anisotropies of the acceptors are, however, determined by the equation:

$r=0.4\left(3 \cos ^{2} \theta-1\right) / 2$

where $\theta$ is the angle between the donor and acceptor transition dipoles. In our case, this angle is $65^{\circ}$ [5]. The anisotropy of the acceptors is, thus, -0.093 , according to Eqn. 6. In summary, we can now write Eqn. 5 as follows:

$r(\infty)=\frac{0.4\left(\sigma_{1}^{2}+\gamma \sigma_{2}^{2}+(1-\gamma) \sigma_{3}^{2}\right)-0.093 \sigma_{2} \sigma_{3}}{\sigma_{1}^{2}+\gamma \sigma_{2}^{2}+(1-\gamma) \sigma_{3}^{2}+\sigma_{2} \sigma_{3}}$

where $\sigma_{1}, \sigma_{2}$ and $\sigma_{3}$ are related to $\beta-155, \alpha-84$ and $\beta-84$, respectively. Using $\gamma=0.43$ and the relative absorption cross-sections at $615 \mathrm{~nm}$, as derived from the published absorption spectra of the individual chro- 
mophores [21,22], we obtain a value of 0.214 for $r(\infty)$. This is in fair agreement with our experimental value of $0.23 \pm 0.02$, although a $60^{\circ}$ angle between dipoles is in better agreement with this value. In order to obtain the direction of the transition dipole moment to a first approximation, the conjugated portion of each chromophore has been fitted to a line by the least-squares method [5]. The real directions may deviate in some extent from these calculated values. We can, thus, conclude that the observed kinetics can be wellaccounted for by Förster-type energy transfer between $\alpha-84$ and $\beta-84$ on the femtosecond time-scale.

In addition, we can calculate the ratio of absorbance change obtained immediately after excitation to its final value, according to the equation:

$$
\begin{aligned}
& \Delta A^{\text {iso }}(0) / \Delta A^{\text {iso }}(\infty) \\
& \quad=\left(\sigma_{1}^{2}+\sigma_{2}^{2}+\sigma_{3}^{2}\right) /\left(\sigma_{1}^{2}+\gamma \sigma_{2}^{2}+(1-\gamma) \sigma_{3}^{2}+\sigma_{2} \sigma_{3}\right)
\end{aligned}
$$

Inserting the appropriate constants, we obtain $\Delta A^{\text {iso }}(0) / \Delta A^{\text {iso }}(\infty)=1.09$, which is in agreement with the small amplitude (10\%) of the fast component in the isotropic kinetic traces (see Fig. 1, curves $\mathrm{c}$ and d). It is difficult, however, to calculate $\Delta A^{\text {iso }}(\infty)$ precisely because slower picosecond processes will influence both the $\Delta A^{\text {iso }}(t)$ and $r(t)$ values. In Fig. 2, a fit with two exponentials with $\tau=0.37$ ps and 30 ps would be in good agreement with the experimental curve. The 370 fs lifetime coincides with the calculated Forster transfer between $\alpha-84$ and $\beta-84$, while the 30 -ps lifetime is similar to the picosecond absorption and fluorescence date reported previously [7-13]. The latter has been interpreted to be due to $\beta-155$ transfer to $\alpha-84(\beta-84)$ [7-9] or as a relaxation between excitonic states [13].

In a recent work on APC trimers [16], we have shown that a 440 -fs isotropic signal is most likely due to Förster-type energy transfer between neighbouring $\alpha-81$ and $\beta-80$ chromophores. In this case, the transfer is supposed to be almost unidirectional, since the chromophores are believed to have different absorption maxima, i.e., about 600 and $650 \mathrm{~nm}$, respectively.

In the case of C-PC, the measured rate is the sum of two rates $\left(K_{\mathrm{DA}}\right.$ and $\left.K_{\mathrm{AD}}\right)$ of approximately equal magnitude, since the $\alpha-84$ and $\beta-84$ chromophores have strongly overlapping spectra [20]. The rate $K_{\text {DA }}$ of excitation-energy transfer from $\alpha-84$ to $\beta-84$ is 1530 $\mathrm{ns}^{-1}$ and corresponds to a 650 -fs process. This is slower than for APC trimers. This result is reasonable, however, because of the expected larger donor-acceptor spectral overlap in the latter case. Detailed calculations of this overlap are still missing, however, since the individual absorption spectra of the $\alpha-84$ and $\beta-84$ chromophores of APC (which has only two different chromophores) are not known at present.

Since the participation of closely-lying excitonic states in the picosecond kinetics has been suggested by several workers $[13,23-25]$, we finally want to discuss this situation. In APC trimers an excitonic coupling between the $\alpha$ - and $\beta$-chromophores has been suggested as an explanation for the shape of the CD-spectrum around $650 \mathrm{~nm}$ [26-29]. An alternative explanation would be that the circular dichroism is due to a specific conformation of one of the chromophores [27]. The CD-spectrum for C-PC trimers does not show the same feature as APC trimers and only a weak excitonic interaction has been suggested to explain for the shape of the CD-spectrum [30,31]. The excitonic coupling between $\alpha-84$ and $\beta-84$ in C-PC trimers calculated from $X$-ray data is $112 \mathrm{~cm}^{-1}$ [23]. Since the coherent spectral width of the pulses used in our experiments was about $300 \mathrm{~cm}^{-1}$, we have not been able to selectively excite a isolated excitonic state, but rather a mixture of two excitonic states. In this case, we should observe the transfer of energy localized on one of the chromophores to the other. In turn, the excitonic splitting is less than the vibrational bandwidths of the electronic states of $\alpha-84$ and $\beta-84$ chromophores. A Förster-type process rather than coherent energy transfer should be observed in this case [32]. Because of the fast loss of coherence, the correction of Förster theory is required for nearly identical excitonically-coupled $\alpha-84$ and $\beta-84$ chromophores only at times as short as tens of femtoseconds [33]. There are, thus, several arguments against the assignment of the observed femtosecond process as a relaxation between excitonic levels. For the APC trimers, the arguments for a 440-fs Förster transfer were recently published [16]. The longer ps components found in both absorption and fluorescence studies of APC trimers were interpreted to be due to distortion of the APC trimer structure upon removal of the linker polypeptide [16]. The 20-30 ps component observed previously in C-PC trimers is, in our opinion, best explained by Förster transfer from $\beta-155$ to the $\alpha-84$ and $\beta-84$ chromophores [7-12,14]. A similar component was also observed in this work in both trimeric and monomeric preparations (Figs. 5 and 6 , respectively).

\section{Acknowledgements}

We thank the Swedish Natural Science Research Council, the Royal Swedish Academy of Sciences, the Russian Academy of Sciences and the Deutsche Forschungsgemeinschaft (SFB 143, project $\mathrm{Al}$ ) for financial support and to Prof. R.S. Knox for fruitful discussions.

\section{References}

1 Whitton, B.A. and Carr, N.G. (1982) in The Biology of Cyanobacteria (Carr, N.G. and Whitton, B.A., eds.), University of California Press, Berkeley. 
2 MacColl, R. and Guard-Friar, D. (1987) Phycobiliproteins, CRC, Boca Raton.

3 Glazer, A.N. (1977) Mol. Cell. Biochem. 18, 124-140.

4 Schirmer, T., Huber, R., Schneider, M., Bode, W., Miller, M. and Hackert, M.L. (1986) J. Mol. Biol. 188, 651-676.

5 Schirmer, T., Bode, W. and Huber, R. (1987) J. Mol. Biol. 196, 677-695.

6 Duerring, M., Schmidt, G.B. and Huber, R. (1991) J. Mol. Biol. 217, 577-592.

7 Wendler, J., John, W., Scheer, H. and Holzwarth, A.R. (1986) Photochem. Photobiol. 44, 79-85.

8 Schneider, S. and Geiselhart, P. (1988) Z. Naturforsch. 43c, $85-92$.

9 Hefferle, P., Geiselhart, P., Mindl, T., Schneider, S., John, W. and Scheer, H. (1984) Z. Naturforsch. 39c, 606-616.

10 Schneider, S., Geiselhart, P., Mindl, T., Hefferle, P., Dörr, F., John, W. and Scheer, H. (1985) in Optical Properties and Structure of Tetrapyrroles (Blauer, G. and Sund, H., eds.), pp. 384-395, Walter de Gruyter, Berlin.

11 Holzwarth, A.R., Wendler, J. and Suter, J.W. (1987) Biophys. J. $51,1-12$.

12 Sandström, Å., Gillbro, T., Sundström, V., Fischer, R. and Scheer, H. (1988) Biochim. Biophys. Acta 933, 42-53.

13 Holzwarth, A.R. (1989) Q. Rev. Biophys. 22, 239-326.

14 Gillbro, T., Sandström, A., Sundström, V., Fischer, R. and Scheer, H. (1988) in Photosynthetic Light-Harvesting Systems (Scheer, H. and Schneider, S., eds.), pp. 457-467, Walter de Gruyter, Berlin.

15 Khoroshilov, E.V., Kryukov, I.V., Kryukov P.G., Sharkov A.V. and Gillbro, T. (1990) Proc. SPIE, Vol. 1403, Laser Applications in Life Sciences, pp. 431-433.

16 Sharkov, A.V., Kryukov, I.V., Khoroshilov, E.V., Kryukov, P.G., Fischer, R., Scheer, H. and Gillbro, T. (1992) Chem. Phys. Lett. 191, 633-638.

17 Füglistaller, P., Suter, F. and Zuber, H. (1983) Hoppe Seyler's Z. Physiol. Chem. 364, 691-712.
18 Fischer, R. and Scheer, H. (1992) J. Photochem. Photobiol. B: Biol. 15, 19-103.

19 Kiyukov, I.V., Kryukov, P.G., Khoroshilov, E.V. and Sharkov, A.V. (1988) Sov. J. Quantum Electron. 18, 830-832.

20 Sauer, K., Scheer, H. and Sauer, P. (1987) Photochem. Photobiol. $46,427-440$.

21 Siebzehnrübl, S., Fischer, R. and Scheer, H. (1987) Z. Naturforsch. 42c, 258-262.

22 Fischer, R., Siebzehnrübl, S. and Scheer, H (1988) in Photosynthetic Light-Harvesting Systems (Scheer, H. and Schneider, S., eds.), pp. 71-76, Walter de Gruyter, Berlin.

23 Sauer, K. and Scheer, H. (1988) Biochim. Biophys. Acta 936, $157-170$.

24 Maxson, P., Sauer, K. and Glazer, A.N. (1988) in Photosynthetic Light-Harvesting Systems (Scheer, H. and Schneider, S., eds.), pp. 439-451, Walter de Gruyter, Berlin.

25 Beck W.F. and Sauer, K. (1992) Biophys. J. 59, 32a.

26 Holzwarth, A.R., Bittersmann, E., Reuter, W. and Wehrmeyer, W. (1990) Biophys. J. 57, 133-145.

27 MacColl, R., Csatorday, K., Berns, D.S. and Traeger, E. (1980) Biochemistry 19, 2817-2832.

28 Canaani, O.D. and Gantt, E. (1980) Biochemistry 19, 2950-2956.

29 Csatorday, K., MacColl, R., Csizmadia, V., Grabowsky, J. and Bagyinka, C. (1984) Biochemistry 23, 6466-6470.

30 John, W., Fischer, R., Siebzehnrübl, S. and Scheer, H. (1985) in Antenna and Reaction Centers of Photosynthetic Bacteria (Michel-Beyerle, M.E., ed.), pp. 17-25, Springer, Berlin.

31 Scharnagl, C. and Schneider, S. (1991) J. Photochem. Photobiol. B: Biol. 8, 129-157.

32 Pope, M. and Swenberg, C.E. (1982) Electronic Processes in Organic Crystals, Oxford University Press, Oxford.

33 Knox, R.S. and Gülen, D. (1993) Photochem. Photobiol. 57, in press. 